\title{
HIGH SPEED STABILITY OF A RAILWAY VEHICLE EQUIPPED WITH INDEPENDENTLY ROTATING WHEELS
}

\author{
O. Kyryl'chuk*, J. Kalivoda**, L. Neduzha***
}

\begin{abstract}
The paper is devoted to the study of lateral oscillation and the assessment of the motion stability of a four axle railway vehicle equipped with wheelsets with independently rotating wheels. First, a linear four degrees of freedom mathematical model of the unconstrained wheelset in a straight track has been built. The wheelset model was consequently utilized in a model of the whole vehicle representing typical railway passenger car. The model has 25 degrees of freedom and it is described by a system of 50 first order homogeneous differential equations. Linear algebra methods were used to assess the stability. The influence of a running velocity, the torsional stiffness of wheelsets and the viscosity of coupling element between wheels was studied. Results of this study shows that wheelsets with independently rotating wheels and viscous coupling element enable stable behaviour of a vehicle at speeds exceeding $500 \mathrm{~km} / \mathrm{h}$. Prospectively, this type of wheelset is applicable to high-speed railway vehicles, because it offers either further increase of running speeds or simplification of running gear while preserving high speed stability.
\end{abstract}

Keywords: railway wheelset, independently rotating wheels, lateral dynamics, stability

\section{Introduction}

Wheelset is a basic technical device providing guiding of railway vehicles. Nearly without exception all railway vehicles, from the beginning of railway transport until now, are equipped with conventional wheelsets (Fig 1, left). Conventional wheelsets are consisted of two wheels firmly fixed to a rigid axle. This forces the both wheels to rotate at the same speed. A wheelset with independently rotating wheels (IRW) is obtained from the conventional one by enabling independent wheel rotation (Fig 1, right). IRW wheelsets are utilized particularly in the construction of low-floor trams in order to lower the floor height in the area above a bogie. Nevertheless, apart from spatial problem solution, IRW wheelsets can offer further advantages.
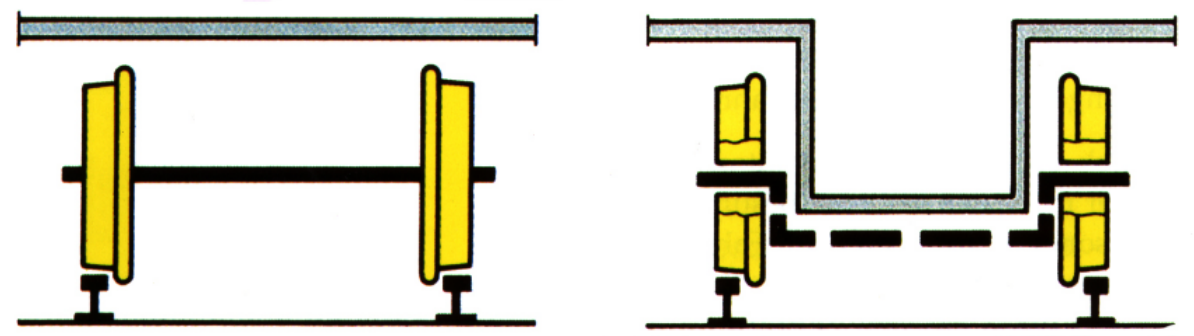

Fig. 1: Conventional and IRW wheelset

\footnotetext{
Oleg Kyryl'chuk: Dnipropetrovsk National University of Railway Transport, Dep. «Car and Car Facilities», Lazaryan St. 2 , 49010, Dnipro, Ukraine, o.a.kirilchuk@gmail.com

** Ing. Jan Kalivoda, PhD.: Czech Technical University in Prague, Faculty of Mechanical Engineering; Technická 4, 16607 Praha 6 CZ, jan.kalivoda@fs.cvut.cz

*** Doc. Larysa Neduzha: Dnipropetrovsk National University of Railway Transport, Dep. «Theoretical and Structural Mechanics», Lazaryan St. 2, 49010, Dnipro, Ukraine, nlorhen@i.ua
} 
Analysing the movement of a conventional wheelset on a rail track it is not difficult to see that it moves not only progressively along the track axis, but also makes lateral movements and rotational movements around its vertical axis. This motion, so called wheelset hunting motion, was first described by Klingel in 1883 (Knothe and Böhm, 1999). While at low running speeds the amplitudes of hunting motion decrease, at high speeds they tend to grow steadily and vehicle movement becomes unstable. The vehicle speed at the limit of stability is called the critical velocity. Since railway vehicles cannot be safely operated at speeds above the critical velocity, is the stability of hunting motion a major limitation of the maximum speed of rolling stock. Todays' railway highs speed vehicles must be equipped by several heavy, complicated and expensive devices (such as yaw dampers) in order to improve stability and increase the critical velocity. At the other hand, once the wheels of a wheelset can rotate independently, the cause of the hunting motion disappears. Thus IRW wheelsets have naturally stable high speed behaviour.

The goal of this paper is to build mathematical model of 4 axle railway passenger car possessing wheelsets with independently rotating wheels, compare its high-speed stability with the same car with conventional wheelsets and assess the influence of elastic and viscous connection between wheelsets' wheels on the railway vehicle stability.

\section{Model of a four-axle railway vehicle}

After composing and analysing a model of unconstrained IRW wheelset (Myamlin et al., 2016) a planar model of four-axle railway vehicle consisting of 7 rigid bodies (car body, two bogie frames and four wheelsets) has been built (Fig. 2). The bodies are connected by viscoelastic linear elements that represents primary and secondary suspension. Tangential forces acting in a wheel rail contacts are determined using Carter's theory (Knothe, 2008)

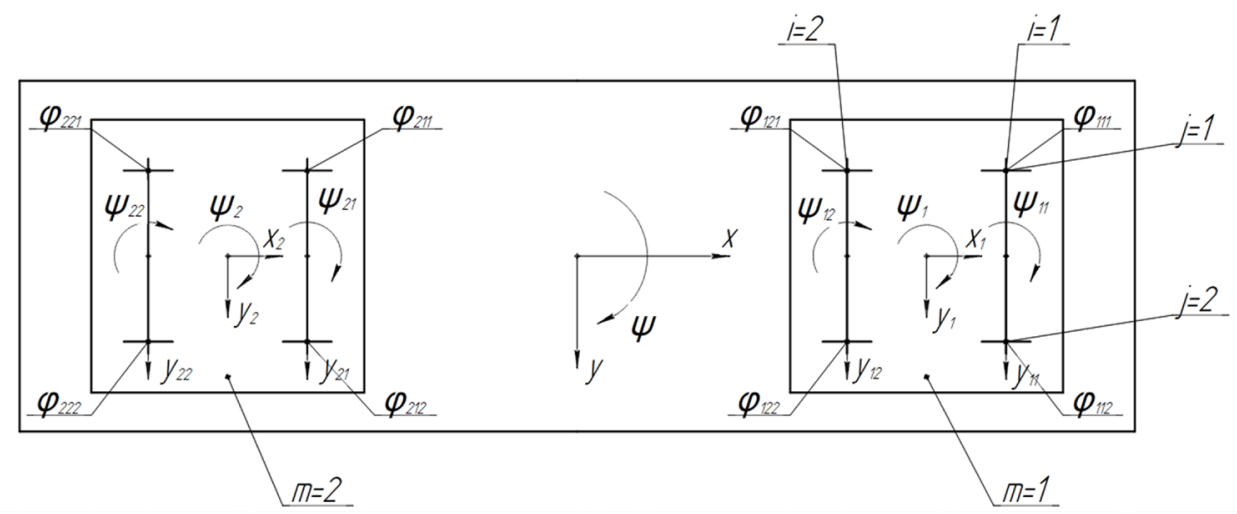

Fig. 2: Plain view of a vehicle model

By expressing the equilibrium of forces for all bodies we obtain a system of 25 linear differential equations of the second order. This system can be written as:

$$
\mathrm{MQ}+\mathrm{BQ}+\mathrm{KQ}=\mathbf{0}
$$

System (1) was then be converted into a system of 50 first-order differential equations in a form

$$
\mathbf{X}=\mathbf{A X}
$$

The coefficients of the matrix A depend not only on vehicle parameters (dimensions, masses, moments of inertia, suspension characteristics) but also on the running velocity. To assess the stability of the motion eigenvalues of matrix $\mathbf{A}$ and the corresponding oscillation increments $\delta$ were calculated. The oscillation increment depends on two consecutive amplitudes of periodic motion according to equation (3).

$$
\delta=\frac{\ln \left(\frac{A(t+T)}{A(t)}\right)}{T}
$$

where $A(t)$ is the amplitude of the oscillation in time $t, A(t+T)$ is the amplitude of the oscillation in time $t+T$ and $T$ is the period of the oscillating motion. The oscillating motion is stable in case of its' amplitudes decrease in time i.e. in the case of negative value of oscillation increment $\delta$. 


\section{Results}

The influence of the running speed, the elasticity of the coupling between the wheels of a wheelset $k_{w}$ and the viscosity of the coupling between the wheels of a wheelset $b_{w}$ were assessed. Figure 3 shows the influence of the elasticity $k_{w}$ on a stability.

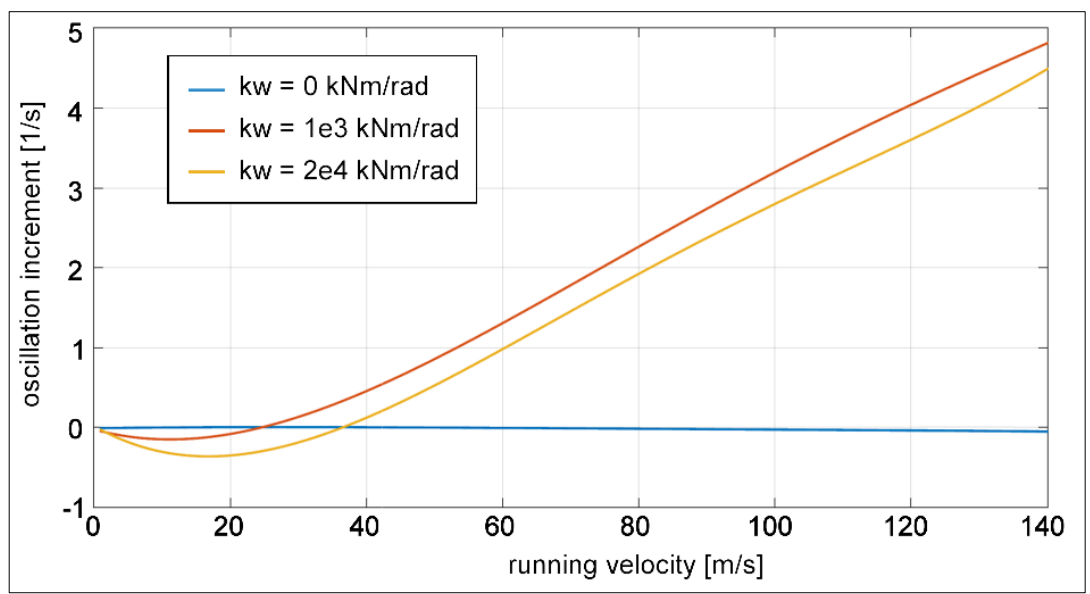

Fig. 3: Dependence of the oscillation increment $\delta$ on the running velocity and elasticity $k_{w}$

Value $k_{w}=0 \mathrm{kNm} / \mathrm{rad}$ represents wheelsets with fully independently rotating wheels (IRW), $k_{w}=2 \cdot 10^{4} \mathrm{kNm} / \mathrm{rad}$ represents conventional solid axle wheelset. Value $k_{w}=1 \cdot 10^{3} \mathrm{kNm} / \mathrm{rad}$ corresponds to the torsionally flexible wheelset.

As it can be seen from the Fig. 3 with elasticity equal to zero the oscillations cease. However, in this case the self centering of the wheelset in the track is also lost. This significantly limits the utilization of IRW wheelsets, since wheelset centering is a prerequisite for ensuring the smooth running of the car and keeping the wear of wheel flanges under acceptable limits. For conventional rigid wheelset we observe a loss of stability at a speed of about $38 \mathrm{~m} / \mathrm{s}(137 \mathrm{~km} / \mathrm{h})$. This is within the range of the critical speed of railway vehicles which the model represents (standard passenger car without yaw dampers). This confirms the adequacy of the model. It is further evident from the Fig. 3 that reducing of the torsional stiffness of wheelsets leads to the increased instability and reduction of critical speed.

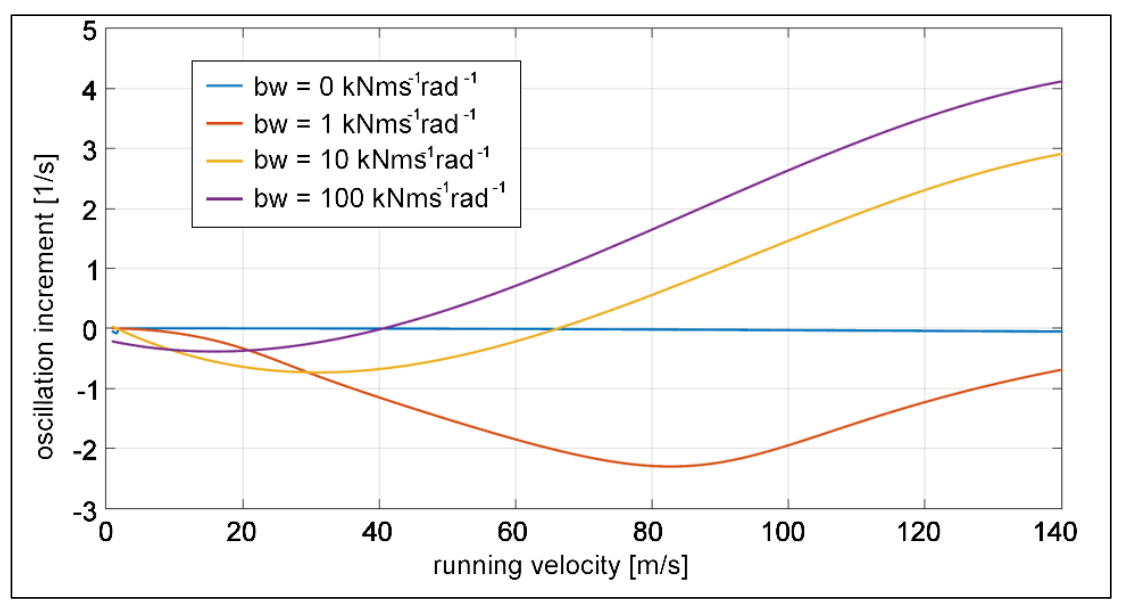

Fig. 4: Dependence of the oscillation increment $\delta$ on the running velocity and viscosity $b_{w}$

On the Fig. 4, we see that in the presence of an element of viscous damping between wheels of a viscosity $b_{w}=1 \mathrm{kNms}^{-1} \mathrm{rad}^{-1}$, it is possible to achieve stable motion at speeds above $140 \mathrm{~m} / \mathrm{s}(500 \mathrm{~km} / \mathrm{h})$. Increasing the viscosity to $b_{w}=10 \mathrm{kNms}^{-1} \mathrm{rad}^{-1}$ leads to a reduction in the critical speed to about $65 \mathrm{~m} / \mathrm{s}$ $(230 \mathrm{~km} / \mathrm{h})$, and finally, the viscosity $b_{w}=100 \mathrm{kNms}^{-1} \mathrm{rad}^{-1}$ almost completely prohibits mutual rotations of the wheels, vehicle reaches a critical velocity about $40 \mathrm{~m} / \mathrm{s}(145 \mathrm{~km} / \mathrm{h})$, i.e. virtually identical value, as for conventional solid axle wheelsets.

A change of the viscosity $b_{w}$ between 0 and $10 \mathrm{kNms}^{-1} \mathrm{rad}^{-1}$ causes in the beginning an increase of the stability and then the stability deterioration. To determine the optimum viscosity of the torsional damping 
element at the velocity of $500 \mathrm{~km} / \mathrm{h}$, plot of the dependence of the oscillation increment on the viscosity $b_{w}$ was calculated (Fig. 5). As can be seen from the graph the maximal stability at the vehicle velocity of $500 \mathrm{~km} / \mathrm{h}$ is reached by the optimal value of the viscosity $b_{w, o p t, 500}=0.32 \mathrm{kNms}^{-1} \mathrm{rad}^{-1}$.

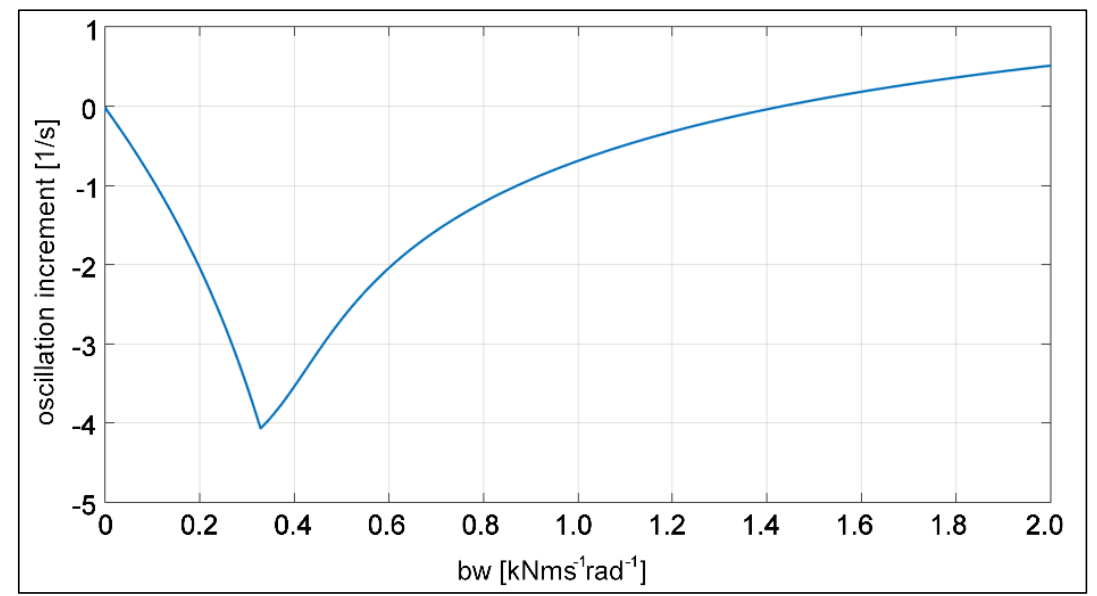

Fig. 5: Dependence of the oscillation increment $\delta$ on the viscosity $b_{w}$ at speed $500 \mathrm{~km} / \mathrm{h}$

It can be shown that the value of the optimum viscosity decreases with increasing speed. However, the viscosity must be high enough to assure natural centering of a wheelset in the track.

\section{Conclusions}

A system of linear differential equations describing lateral dynamics of railway vehicle was formulated. Based on the calculation of eigenvalues and the oscillation increment of the system a high-speed stability of railway vehicle was assessed. The results confirm significant increase in a critical velocity of a vehicle with IRW wheelsets compared to a conventional one. However, wheelsets with purely independent wheel rotation lose the ability to naturally center towards a track. Therefore, various types of coupling between the wheels of wheelset were examined. The goal of such coupling is to provide wheelset centering, whilst preserve high-speed stability of IRW wheelset. It has been found that elastic coupling between wheels causes a reduction in stability and critical speed. Nevertheless, the viscous coupling appears to be promising. The optimum viscosity value, which provides stable wheelset oscillations even at speeds of $500 \mathrm{~km} / \mathrm{h}$, can be found. However, those results were obtained by linear model that does not fully respect all the phenomena associated with the rolling stock moving at high speeds. Experimental verification is required. Because track tests are financially and organizationally extremely demanding, utilization of the CTU roller rig (Myamlin et al., 2017, Kalivoda and Bauer, 2016) is planned in order to verify the simulation results described above.

\section{Acknowledgement}

This paper was created with the support of Technology Agency of the Czech Republic, project No TE01020038 “Competence Centre of Railway Vehicles".

\section{References}

Kalivoda, J. and Bauer, P. (2016) Mechatronic bogie for roller rig tests. The Dynamics of Vehicles on Roads and Tracks, Proceedings of the $24^{\text {th }}$ Symposium of the International Association for Vehicle System Dynamics, pp. 899-908. DOI: 10.1201/b21185-96.

Knothe, K. (2008) History of wheel/rail contact mechanics: from Redtenbacher to Kalker. Vehicle System Dynamics, Vol. 46, Issue 1-2, pp. 9-26.

Knothe, K. and Böhm, F. (1999) History of Stability of Railway and Road Vehicles. Vehicle System Dynamics, Vol. 31, Issue 5-6, pp. 283-323.

Myamlin, S., Kalivoda, J. and Neduzha, L. (2017) Testing of Railway Vehicles Using Roller Rigs. Procedia Engineering. Vol. 187 (2017), pp. 688-695. DOI: 10.1016/j.proeng.2017.04.439.

Myamlin, S., Kirilchuk, O. and Metyzhenko, V. (2016) Mathematical model of wheelset oscillations with independent wheel rotation in the horizontal plane. Science and transport progress. № 4 (64), pp. 134-141, DOI: $10.15802 /$ stp2016/77999. 